Periodica Mathematica Hungarica Vol. 2 (1-4), (1972), pp. 15-19.

\title{
TOTALLY DISCONNECTED SETS, JORDAN CURVES, AND CONFORMAL MAPS ${ }^{1}$
}

\author{
by \\ G. PIRANIAN (Ann Arbor) \\ Dedicated to the memory of ALFRÉD RÉNYI
}

Each bounded, closed, totally disconnected set $M$ in the $w$-plane lies on some Jordan curve $\Gamma$ (see R. L. Moore and J. R. KLINE [3]). Let $G$ denote the bounded domain determined by $\Gamma$, let $D$ and $C$ denote the unit disk and the unit circle in the z-plane, and let $f$ be a mapping of $D \cup C$ onto $G \cup I$, holomorphic in $D$ and continuous and univalent in $D \cup C$. Kikuji Matsumoto ([2], Theorem 4) showed that if we pinch the domain $G$ in appropriate places, then the set $f^{-1}(M)$ has logarithmic capacity 0 . In this note, we prove that we can not only make the set $f^{-1}(M)$ arbitrarily thin, but that we can require it to lie in any preassigned perfect subset of $C$.

Theorem. Let $M$ be a bounded, closed, totally disconnected set in the wplane, and let $E$ be a perfect set on $C$. Then there exists a function $f$, holomorphic in $D$ and continuous and univalent in $D \cup C$, such that $f^{-1}(M) \subset E$.

Our proof is based on the construction of a certain tree $T$ and a certain Jordan domain $G_{0}$ in the $w$-plane. The tree lies in $G_{0}$, and the derived set of its set of vertices is $M$. A simple analytic process allows us to replace the tree $T$ with a subdomain $G$ of $G_{0}$ such that one of the corresponding holomorphic and univalent functions $f$ from $D \cup C$ onto $\bar{G}$ satisfies the condition $f(E) \supset M$.

The tree. Without loss of generality, we may assume that the set $M$ lies in the open rectangle $Q$ whose vertices are the points $w= \pm \sqrt{2} / 2$ and $w=$ $= \pm \sqrt{2} / 2+i$. Since $M$ is closed and totally disconnected, there exists a directed polygonal arc $P$ that begins at the point 0 , lies in $Q \backslash M$, and separates $Q$ into two components $Q_{0}$ and $Q_{1}$, each of diameter less than $\sqrt{3}(4 / 5)$. Similarly, there exist two directed polygonal ares $P_{0}$ and $P_{1}$ in $Q_{0} \backslash M$ and $Q_{1} \backslash M$, with a common initial point on $P$, and such that each of the four corresponding sets $Q_{00}, Q_{01}, Q_{10}, Q_{11}$ has diameter less than $\sqrt{3}(4 / 5)^{2}$. We continue the dissec-

1 This paper was written with support from the National Science Foundation. 
tion of $Q$ indefinitely, in such a way that each polygonal are of the $n^{\text {th }}$ stage is divided into two parts by the common initial point of two ares of the $(n+1)^{\text {st }}$ stage. The union of the anterior parts thus determined constitutes a tree $T_{0}$, and each vertex of $T_{0}$ (except the point $w=0$ ) has degree 2 or 3 (see the heavily drawn portion of Figure 1). We may assume that the directions of two consecutive segments of $T_{0}$ always differ by less than $\pi / 2$.

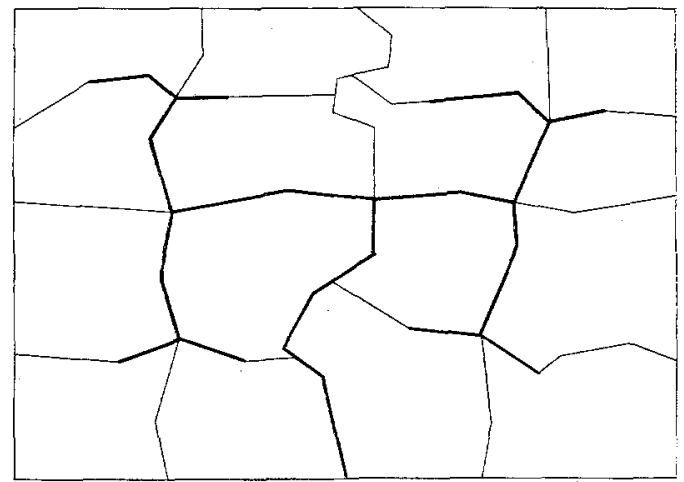

Fig. 1

Because the set $M$ meets none of the polygonal arcs $P, P_{0}, P_{1}, P_{0 G}$, $P_{01}, \ldots$, each point of $M$ is the limit point of exactly one simple path that begins at 0 and lies in $T_{0}$. The union of all simple paths beginning at 0 , lying in $T_{0}$, and having a limit point in $M$ constitutes our tree $T$.

The domain $G_{0}$. We arrange the segments of $T$ into a sequence $\left\{S_{m}\right\}$ so that $m_{0}<m$ whenever $S_{m_{0}}$ precedes $S_{m}$ in $T$, and so that $\left|m_{0}-m\right|=1$ whenever $S_{m_{0}}$ and $S_{m}$ have a common initial point. We then choose a sequence $\left\{\delta_{m}\right\}$ of positive numbers, and we denote by $H_{m}$ the set of all points whose distance from $S_{m}$ is less than $\delta_{m}$. If $\delta_{m} \rightarrow 0$ rapidly enough, then the set $G_{0}=\bigcup H_{m}$ is a Jordan domain, and for each index $m$ the intersection of $M$ with the closure of $H_{m}$ is empty.

The analytic device. Barring an obvious geometric obstacle, the following lemma allows us to pass from any univalent function $f$ in $|z|<r_{0}\left(r_{0}>1\right)$ to a univalent function $g$ such that the essential difference between the domains $f(D)$ and $g(D)$ is a narrow rod of prescribed base, length, and direction.

Lemma (compare [1], pp. 43-44). Suppose that the function $f$ is holomorphic and univalent in some disk $|z|<r_{0}\left(r_{0}>1\right)$. Let $\zeta=e^{i \theta}$, and let $L$ be a complex number such that

$$
\left|\arg L-\arg \zeta f^{\prime}(\zeta)\right|<\pi / 2
$$


and such that the line segment $S$ joining the points $f(\zeta)$ and $f(\zeta)+L$ meets the set $f(D \cup C)$ only at $f(\zeta)$. Corresponding to each real number $\varrho(\varrho<1)$, write

$$
g_{e}(z)=f(z)+L \frac{\log \left(1-z / z_{0}\right)}{\log (1-1 / \varrho)},
$$

where $z_{0}=\varrho^{i \theta}$. Then there exists a constant $\varrho_{0}\left(\varrho_{0}>1\right)$ such that for $1<\varrho<\varrho_{0}$ the function $g_{\varrho}$ is univalent in some disk $|z|<r_{1}\left(1<r_{1}<\varrho\right)$.

To prove the lemma, we write $\varrho=1+\varepsilon$, we impose the preliminary restrictions $\varepsilon<1 / e$ and $\varepsilon<\left(r_{0}-1\right) / 2$, and we observe that the univalence of $f$ in $|z|<r_{0}$ implies the existence of a positive constant $A_{1}$ such that the inequality

$$
\left|f\left(z_{2}\right)-f\left(z_{1}\right)\right| \geqq A_{1}\left|z_{2}-z_{1}\right|
$$

holds for all $z_{1}$ and $z_{2}$ in $D \cup C$. We write $z / \zeta=\alpha+i \beta$ ( $a$ and $\beta$ real), and we consider the function $g_{e}$ separately in the two overlapping regions

$$
\begin{aligned}
& D_{1}=\{z:|z| \leqq 1, \alpha \leqq 1-K|| \log \varepsilon \mid\}, \\
& D_{2}=\left\{z:|z| \leqq 1, \alpha \geqq 1-(\log |\log \varepsilon|)^{-1}\right\}
\end{aligned}
$$

(see Figure 2); here $K$ denotes a positive number to be chosen below.

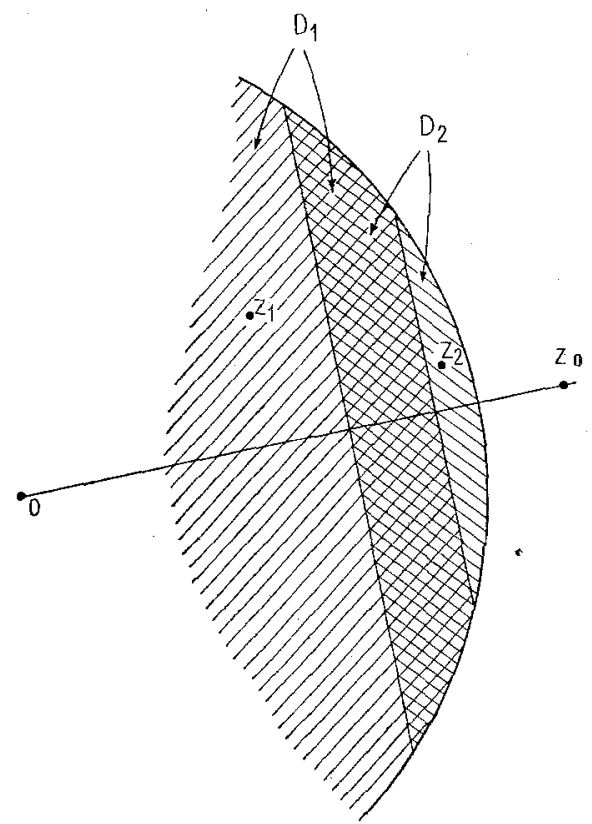

Fig. 2 
Since

$$
g_{\varrho}^{\prime}(z)-f^{\prime}(z)=\frac{L}{|\log \varepsilon| \varrho \mid\left(z-z_{0}\right)},
$$

and since in $D_{1}$ the maximum modulus of the right-hand member is

the inequality

$$
\frac{|L|}{|\log \varepsilon / \varrho|(\varepsilon+K /|\log \varepsilon|)}<|L| / K,
$$

$$
\left|g_{\varrho}\left(z_{2}\right)-g_{\varrho}\left(z_{1}\right)\right| \geq\left|z_{2}-z_{1}\right|\left(A_{1}-|L| / K\right)
$$

holds for all $z_{1}$ and $z_{2}$ in $D_{1}$. In particular, the choice $K=A_{1} / 2|L|$ gives the inequality

$$
\left|g_{\varrho}\left(z_{2}\right)-g_{\varrho}\left(z_{1}\right)\right| \geqq A_{1}\left|z_{2}-z_{1}\right| / 2
$$

and therefore $g_{0}$ is univalent in $D_{1}$.

To establish univalence in $D_{2}$, we examine the argument of the derivative

$$
g_{\varrho}^{\prime}(z)=\frac{1}{z_{0}}\left[z_{0} f^{\prime}(z)+\frac{L}{|\log \varepsilon / \varrho|} \cdot \frac{1}{\left(1-z / z_{0}\right)}\right] \text {. }
$$

By the inequality (1), the argument of the first term in the brackets is restricted to some interval $[\arg L-\eta, \arg L+\eta]$, where $\eta<\pi / 2$ if $\varepsilon$ is sufficiently small. Because the argument of the second term is also restricted to such an interval, the theorem of K. NosHrRo and S. E. WARSCHAWsKI implies that the function $g_{9}$ is univalent in $D_{2}$ (see [4], Theorem 12, p. 151; [5], Lemma 1, p. 312).

To conclude the proof of the lemma, we shall show that if $z_{1} \in D_{1} \backslash D_{2}$ and $z_{2} \in D_{2} \backslash D_{1}$, then $g_{0}\left(z_{1}\right)$ lies at a greater distance from the segment $S$ than $g_{\varrho}\left(z_{2}\right)$.

Our hypothesis on the line segment $S$ implies the existence of a positive constant $A_{2}$ such that for each $z$ in $D \cup C$ the distance between $f(z)$ and the segment $S$ is at least $A_{2}|z-\zeta|$. Therefore the distance between $f\left(z_{1}\right)$ and the segment $S$ is at least $A_{2}(\log |\log \varepsilon|)^{-1}$. Since the imaginary part of $\log \left(1-z / z_{0}\right)$ is bounded by $\pi / 2$, the distance between $g_{\varrho}\left(z_{1}\right)$ and $S$ is at least

$$
A_{2}(\log |\log \varepsilon|)^{-1}-2|L| \cdot|\log \varepsilon|^{-1}>A_{3}(\log |\log \varepsilon|)^{-1} \text {. }
$$

On the other hand, (2) implies that if $A_{4}$ denotes the maximum modulus of $f^{\prime}$ on $C$, then the distance between $g_{\varrho}\left(z_{2}\right)$ and $S$ is less than

$$
A_{4} \sqrt{2 K /|\log \varepsilon|}+\frac{|L| \pi / 2}{|\log \varepsilon| \varrho \mid}<A_{5} / \sqrt{|\log \varepsilon|} .
$$

This shows that $g_{Q}\left(z_{1}\right) \neq g_{0}\left(z_{2}\right)$, and the lemma is proved. 
Construction of the domain $G$. We choose any point $z_{1}$ in the perfect set $E$, and we denote by $L_{1}$ the coordinate of the endpoint of the segment $S_{1}$ in the tree $T$. If $\underline{Q}_{1}-1$ is small enough, then the function

$$
f_{1}(z)=L_{1} \cdot \frac{\log \left(1-z / \varrho_{1} z_{1}\right)}{\log \left(1-z / \varrho_{1}\right)}
$$

maps the set $D \cup C$ onto a region lying in $H_{1}$ and containing the segment $S_{1}$.

If $L_{1}$ is not a branch point of the tree $T$, we write $z_{2}=z_{1}$, and we construct the function

$$
f_{2}(z)=f_{1}(z)+L_{2} \frac{\log \left(1-z / \varrho_{2} z_{2}\right)}{\log \left(1-1 / \varrho_{2}\right)}
$$

choosing $L_{2}$ so that $f_{2}$ maps $z_{2}$ onto the endpoint of $S_{2}$, and choosing $\varrho_{2}$ near enough to 1 so that $f(D \cup C) \subset H_{1} \cup H_{2}$. If $L_{1}$ is a branch point of $T$, we choose two distinct points $z_{2}$ and $z_{3}$ of $E$ near $z_{1}$ (this is possible, since $E$ is perfect), and we construct the function $f_{3}$ so that

$$
f_{3}(D \cup C) \subset H_{1} \cup H_{2} \cup H_{3},
$$

and so that $f_{3}\left(z_{2}\right)$ and $f_{3}\left(z_{3}\right)$ are near enough to the endpoints of $S_{2}$ and $S_{3}$ to allow the obvious continuation of the process.

Clearly, the function $f=\lim f_{m}$ is univalent and continuous in $D \cup C$, and $f(D) \subset G_{0}$. Since $E$ is closed and each point of $M$ is a limit point of the sequence $\left\{f\left(z_{m}\right)\right\}$, the set $M$ lies in the set $f(E)$. This concludes the proof of the theorem.

If we drop the hypothesis that the set $M$ is bounded, the theorem remains valid provided we interpret continuity in terms of the spherical metric.

\section{REFERENCES}

[1] F. Herzog and G. Piraniax, Sets of convergence of Taylor series. II, Duke Math. $J .20$ (1953), $41-54$.

[2] K. Matsunoto, On some boundary problems in the theory of conformal mappings of Jordan domains, Nagoya Math. J. 24 (1964), 129-141.

[3] R. L. Moore and J. R. KITNE, On the most general plane closed point-set through which it is possible to pass a simple continuous aro, Ann. of Main. (2) 20 (1918$19), 218-223$.

[4] K. Noshrro, On the theory of schlicht functions, J. Fac. Sci. Holkaido Univ. Ser. I $2(1934-35), 129-155$.

[5] S. E. WARSCHAWSKI, On the higher derivatives at the boundary in conformal mappings, Trans. Amer. Math. Soc. 38 (1935), 310-340.

(Received August 23, 1970)

DEPARTMEXT OF MATHEMATICS

UNIVERSITY OF MICHIGAN

3220 ANGELT, HALL,

ANN ARBOR, MICHIGAY 48104

U.S.A. 